\title{
Le regard des photographes commerciaux. Quelques clichés du fonds égyptien de la Collection Fouad Debbas à l'étude.
}

The gaze of commercial photographers. Some pictures of the Egyptian Fouad Debbas Collection of the study

Yasmine Chemali et Anne-Hélène Perrot

\section{(2) OpenEdition}

Journals

Édition électronique

URL : http://journals.openedition.org/cel/406

DOI : $10.4000 /$ cel. 406

ISSN : 2262-208X

Éditeur

École du Louvre

Édition imprimée

Date de publication : 1 octobre 2014

Référence électronique

Yasmine Chemali et Anne-Hélène Perrot, « Le regard des photographes commerciaux. Quelques clichés du fonds égyptien de la Collection Fouad Debbas à l'étude. », Les Cahiers de l'École du Louvre [En ligne], 5 | 2014, mis en ligne le 01 octobre 2014, consulté le 01 mai 2019. URL : http:// journals.openedition.org/cel/406 ; DOI : 10.4000/cel.406

Les Cahiers de l'École du Louvre sont mis à disposition selon les termes de la licence Creative Commons Attribution - Pas d'Utilisation Commerciale - Pas de Modification 4.0 International. 


\title{
Cahiers de l'École du Louvre
} recherches en histoire de l'art, histoire des civilisations archéologie, anthropologie et muséologie

\section{Numéro 5. Octobre 2014}

\author{
Le regard des photographes commerciaux. \\ Quelques clichés du fonds égyptien \\ de la Collection Fouad Debbas à l'étude \\ Yasmine Chemali - Anne-Hélène Perrot
}

Article disponible en ligne à l'adresse :

http://www.ecoledulouvre.fr/cahiers-de-l-ecole-du-louvre/numero5octobre2014/ChemaliPerrot.pdf

Pour citer cet article :

Yasmine Chemali, Anne-Hélène Perrot, «Le regard des photographes commerciaux. Quelques clichés du fonds égyptien de la Collection Fouad Debbas à l'étude ", Cahiers de l'École du Louvre. Recherches en histoire de l'art, histoire des civilisations, archéologie, anthropologie et muséologie [en ligne] n 5 , octobre 2014, p. 47 à 57.

\section{cc) creative \\ ㅂ.: $\ominus \$$}

(C) École du Louvre

Cet article est mis à disposition selon les termes de la Licence Creative Commons Attribution - Pas d'utilisation commerciale - Pas de modification 3.0 non transposé. 


\section{Cahiers de l'École du Louvre recherches en histoire de l'art, histoire des civilisations archéologie, anthropologie et muséologie \\ Numéro 5. octobre 2014}

\section{Sommaire}

\section{Introduction}

L'archéologie en construction : objets, images, dispositions

Nathan Schlanger

La collection de vases grecs du marquis de Northampton (1790-1851).

Entre archéologie et sciences de la nature

Marie-Amélie Bernard.

L'artisanat touristique du Sud-Ouest des États-Unis. L'exemple des objets collectés par Alphonse Pinart à Santa Fe, à la fin du XIX ${ }^{\mathrm{e}}$ siècle Éloïse Galliard

Regard sur les statuettes hindoues et bouddhiques en bronze d'Indonésie. Leur rôle pour la connaissance de la civilisation javanaise ancienne et ses liens avec l'Asie du Sud et du Sud-Est

Mathilde Mechling 25-33

Le Tigre, le Louvre et l'échange de connaissances archéologiques visuelles entre la France et la Grande-Bretagne aux alentours de 1850

Mirjam Brusius p. $34-46$

Le regard des photographes commerciaux. Quelques clichés du fonds égyptien de la Collection Fouad Debbas à l'étude.

Yasmine Chemali - Anne-Hélène Perrot

Émile Guimet et la morsure du canard égyptien.

Un curieux au musée de Boulaq

Thomas Lebée

L'exposition préhistorique de la Galerie de l'Histoire du travail en 1867. Organisation, réception et impacts 


\section{Yasmine Chemali - Anne-Hélène Perrot}

Fouad César Debbas (1930-2001) fut l'un des grands collectionneurs de photographies et autres images sur l'Orient. En 1975, alors ingénieur diplômé de l'École Centrale, c'est en se promenant sur les quais de Seine qu'il découvre un lot de cartes postales anciennes de son pays d'origine, le Liban. Au fil des ans, il poursuit sa collecte en s'intéressant au patrimoine de son pays mais également aux images de l'ensemble des pays d'Orient. Aujourd'hui conservée à Beyrouth, sa collection rassemble environ 35000 photographies, tous supports confondus - tirages sur papier, albums photographiques, vues stéréoscopiques, cartes de visite, cartes postales, négatifs verres ou souples, gravures, journaux - couvrant plus d'un siècle de production d'images des années 1830 aux années 1950. Plus qu'un simple collectionneur, Fouad Debbas a également publié plusieurs ouvrages de référence sur la photographie et la carte postale consacrés au Liban ${ }^{1}$, ainsi qu'aux studios photographiques installés dans la région, dont celui du français Félix Bonfils². Ses recherches ont portés sur les familles, les monuments et les différents quartiers des grandes villes, les universités se créant au XIX ${ }^{e}$ siècle à Beyrouth, les studios photographiques, les cafés ou encore les restaurants.

Si le collectionneur libanais s'est avant tout passionné pour son pays, l'Égypte, étape essentielle du Grand Tour en Orient, est également bien représentée parmi les tirages de la collection. Les images du pays font partie intégrante des albums des premiers photographes installés dans la région, parfois accompagnée de clichés de la Syrie, de la Palestine ou encore de la Grèce.

Alors que « l'Égyptomanie » prend ses racines beaucoup plus tôt ${ }^{3}$, la campagne d'Égypte en 1798, puis les collections rassemblées par les consuls dans les années 1820 ont contribué au développement de la fascination des Européens pour l'Égypte ancienne tout au long du XIX siècle. La participation remarquée de l'Égypte à l'Exposition universelle de 1862 ainsi qu'à celles qui suivirent permettent au public d'observer des antiquités mais également d'avoir un aperçu du pays moderne. Promesse de vestiges antiques et de dépaysement, l'Égypte s'offre, dès lors, comme une destination de prédilection pour les voyageurs. Grâce à l'avancée technique de la navigation à vapeur, les "voyages organisés " de Thomas Cook se développent dès 1869, année qui voit également l'inauguration du canal de Suez. En quête d'aventure, ces Cooks et ces Cookesses ${ }^{4}$ - composés de roturiers, classes bourgeoises, et élites fortunées - sont animés d'une soif de découverte d'un espace désormais élargi à l'ensemble du bassin méditerranéen.

1. Fouad Debbas, Beirut Our Memory, A guided tour illustrated with postcards from the collection of Fouad Debbas, Beyrouth, 1986. Fouad Debbas, Le Mont Liban : 60 reproductions de photographies anciennes, Paris, 1996. Fouad Debbas, Des photographes à Beyrouth : 18401918. Paris, 2001. Voyages en Orient, journal de la Comtesse de Perthuis, 1853-1855, 18601862, manuscrit inédit découvert par Fouad Debbas, Beyrouth, éditions Dar An-Nahar, 2007 (ouvrage posthume publié d'après les recherches de Fouad Debbas).

2. Cette importante partie de la collection fait l'objet d'une numérisation dans le cadre d'une bourse attribuée par le programme « Archives en danger » de la British Library financé par Arcadia : EAP 644: http://eap.bl.uk/database/results.a4d?projlD=EAP644: $r=41$

3. Jean-Marcel Humbert, L'Égyptomanie dans l'art occidental, Paris, 1989, pp. 10-18. II donne, en outre, de l'Égyptomanie la définition suivante: «concept qui recouvre toutes les réutilisations d'éléments décoratifs et de thèmes empruntés à l'Égypte ancienne dans des formes et des objets variés, sans rapport avec leur utilisation et leur raison d'être d'origine ».

4. Sandrine Gamblin, "Thomas Cook en Égypte et à Louxor : I'invention du tourisme moderne au XIXe siècle », Téoros, 25-2 | 2006, pp. 19-25. 
Conséquence de cet essor touristique, dès 1860 la photographie dite commerciale se développe. Rapidement suivie par l'apparition de la carte postale durant les années 1880, la photographie amateur, va, quant à elle, banaliser peu à peu la production professionnelle 5 . L'étude de Nissan Perez, couvrant la période 1839-1885, répertorie 250 photographes ; parmi eux, beaucoup sont des hommes européens ${ }^{6}$. Certains, tout en conservant leur studio dans leur pays d'origine, parcourent les différentes régions, comme Francis Frith ${ }^{7}$, tandis que d'autres choisissent d'implanter leur atelier en Orient. Itinérants ou résidents, ces photographes répondent à la demande d'une clientèle composée de voyageurs ou de simples curieux européens ${ }^{8}$.

Les tirages sont diffusés sous divers formats - tels quels, contrecollés sur support cartonné ou reliés dans de luxueux albums - puis sont proposés à la vente dans les ateliers des photographes ou distribués par des agences, comme Giraudon à Paris ou Mansell à Londres`. Grâce aux nouveaux procédés d'impression, on les retrouve également sous la forme de recueil de planches.

La photographie, dès son origine, recèle un paradoxe : le public reçoit ces images comme une vérité immortalisée, alors que la démarche du photographe implique une part d'artifice, de théâtralisation, de mise en scène ${ }^{10}$. Artisans subjectifs, les premiers photographes professionnels se donnent pour mission d'offrir à l'Occident une vision souvent exotique de l'Orient reçu comme un monde stéréotypé et codifié ${ }^{11}$. Certains d'entre eux s'installent dans la région - et notamment en Égypte - dans ce but. Lallemand Wilhelm Hammerschmidt ${ }^{12}$ est au Caire dès 1860 : il y crée un magasin dans le quartier du Mûski ${ }^{13}$ où il vend ses images ainsi que des produits et des équipements photographiques ${ }^{14}$. Au Caire également, Émile Béchard ${ }^{15}$ ouvre un atelier de portrait dans le quartier de l'Azbakiyya. Hippolyte Arnoux ${ }^{16} s^{\prime}$ établit à Port-Saïd dans les années 1860 tandis qu'Antonio Beato ${ }^{17}$, après un bref passage au Caire, s'installe à Louxor en 1864. Quelques années plus tard, Gabriel Lékégian ${ }^{18}$ au Caire et les frères Zangaki ${ }^{19}$ à Port-Saïd représentent la seconde génération de photographes commerciaux installés en Égypte. La famille Bonfils, quant à elle, ouvre son atelier à Beyrouth en 1867, mais possède très vite une branche au Caire tandis que les Sebah ${ }^{20}$ ou les frères Abdullah s'installent à Constantinople tout en ouvrant des succursales en Égypte.

Dans une logique davantage commerciale que documentaire, les antiquités sont beaucoup photographiées, et l'on retrouve les plus célèbres monuments

5. Cette banalisation est la conséquence de la découverte des propriétés du gélatino-bromure d'argent, voir Anne Lacoste "Photographie et égyptologie, Émile Prisse d'Avennes précurseur (ou pas)?", pp. 29-46 dans Mercedes Volait, Émile Prisse d'Avennes ; Un artiste-antiquaire en Égypte au XIXe siècle, BdE 156, IFAO, 2013, p. 46.

6. Nissan Perez, Focus East. Early Photography in the Near East, 1839-1885, Jerusalem, The Domino Press Ltd., 1988, pp. 124-233.

7. N. Perez, op. cit. note 6,1988, p. 97.

8. Sylvie Aubenas, «Les photographes en Orient, 1850-1880 » Sylvie Aubenas, Jacques Laccarière, Voyage en Orient, Paris, Bibliothèque nationale de France, 2001, p. 25.

9. Lionel Gauthier, “Femmes orientales et photographes commerciaux. La construction d'un imaginaire dans la production photographique des années 1860-1880 », CyberGEO, 2011, p. 3.

10. Édouard Charton, "Préface », Le Tour du Monde, vol.1, V-VIII, 1860. Pour Edouard Charton fondateur des revues Le Magasin Pittoresque, L'Illustration et Le Tour du Monde, la photographie « est un miroir dont le témoignage matériel ne saurait être suspect » (1860, p.VII). cité par L. Gauthier, op. cit. note 9, p. 3.

11. Sur l'exotisme, voir Jean-François Staszac, « Qu'est-ce que l'exotisme? », Le Globe, t. 148 2008, pp. 7-30. Nous retiendrons ici la définition de l'exotisme qui est donnée dans le résumé de son article " L'exotisme n'est pas le propre d'un lieu ou d'un objet mais d'un point de vue et d'un discours sur ceux-ci [...]”

12. Vers 1830 - après 1869.

13. Hélène Bocard, «l'époque des amateurs : 1839-186 dans Mercedes Volait, Le Caire dessiné et photographié au XIXe siècle, Paris, Picard, CNRS, INHA, 2013, p. 175.

14. Alain D'Hooghe dir., Les trois grandes égyptiennes, Les pyramides de Gizeh à travers l'histoire de la photographie, Paris, Marval, 1996, p. 21.

15. Actif entre 1870 et vers 1880. Son frère, Hippolyte Béchard, était quant à lui installé à la Grand-Combe dans le Gard et s'occupait du tirage et de la diffusion en Europe de la production de son frère. Thomas Cazentre, "Photographes du Caire dans le dernier tiers du XIXe siècle : les ateliers commerciaux 》 dans op. cit. note 13, pp. 232-234.

16. Actif entre 1860 et 1890 .

17. Vers 1835-1906.

18. Actif entre 1887 et 1925 .

19. Georges et Constantin Zangaki, actifs entre 1870 et 1915.

20. Le fondateur Pascal Sebah (1823-1886) crée son atelier à 1857 à Constantinople, ouvre une succursale au Caire en 1873 ; son atelier sera repris par son fils Jean (qui signait Jean-Pascal Sebah) en 1888. Il s'associera un temps à Polycarpe Joaillier et ils signeront tous deux une importante production « Sebah \& Joaillier ». La succursale du Caire ferme entre 1895 et 1905, au départ de Joaillier. T. Cazentre, art. cit. note 15, pp. 238-240. 
égyptiens dans la production de ces photographes. Le pays moderne et notamment l'architecture, sont également bien représenté, ainsi que la population, largement mise en scène dans les clichés. Les tirages sont le plus souvent légendés et signés même si la paternité de certains d'entre eux reste parfois discutée ; il n'était pas rare, en effet, que les photographes pratiquent des échanges de clichés afin d'étoffer leurs catalogues.

Répondant à la demande européenne, ce type de production photographique donne à voir un Orient merveilleux et idéalisé auquel le médium confère une aura réaliste. Toutefois, après une étude attentive des clichés, l'effet de réel dont bénéficie le rendu de la photographie s'atténue et laisse entrevoir des approches différant d'un photographe à l'autre. L'observation de ces photographies, facilitée par le cadre de la collection, nous permet de constater que les compositions évoluent, entre mise en scène et sensibilité artistique.

\section{Le rapport à la monumentalité}

Dans la photographie du XIXe siècle, le rapport à la monumentalité s'exprime à la fois par la mise à l'échelle - grâce à un figurant - des monuments ou des sites photographiés, mais aussi par une recherche de la verticalité. À celui qui ne l'a pas vécue, le photographe tâche de transmettre la sensation d'immensité à laquelle il se trouve confronté. Les prises de vues des pyramides au nord et des temples égyptiens au sud, comme Médinet Habou ${ }^{21}$, les célèbres " colosses de Memnon " ${ }^{22}$ ou Abou Simbel ${ }^{23}$, sont autant d'occasion de la restituer.

Les photographes professionnels en expédition en Égypte étaient souvent accompagnés de guides, d'interprètes ou même de simples porteurs qu'ils utilisent comme figurants pour donner l'échelle ou ajouter du pittoresque ${ }^{24}$. Maxime Du Camp raconte: "Toutes les fois que j'allais visiter des monuments, je faisais apporter avec moi des appareils de photographie et j'emmenais un de mes matelots nommé Hadji-Ismaël. C'était un fort beau Nubien ; je l'envoyais grimper sur les ruines que je voulais reproduire, et j'obtenais ainsi une échelle de proportion toujours exacte ${ }^{25} . »$ Cette habitude prise par Du Camp lors de sa mission sera conservée quelques années plus tard par la génération des photographes commerciaux, comme Beato, Bonfils et Sébah" ${ }^{26}$ (fig. 1).
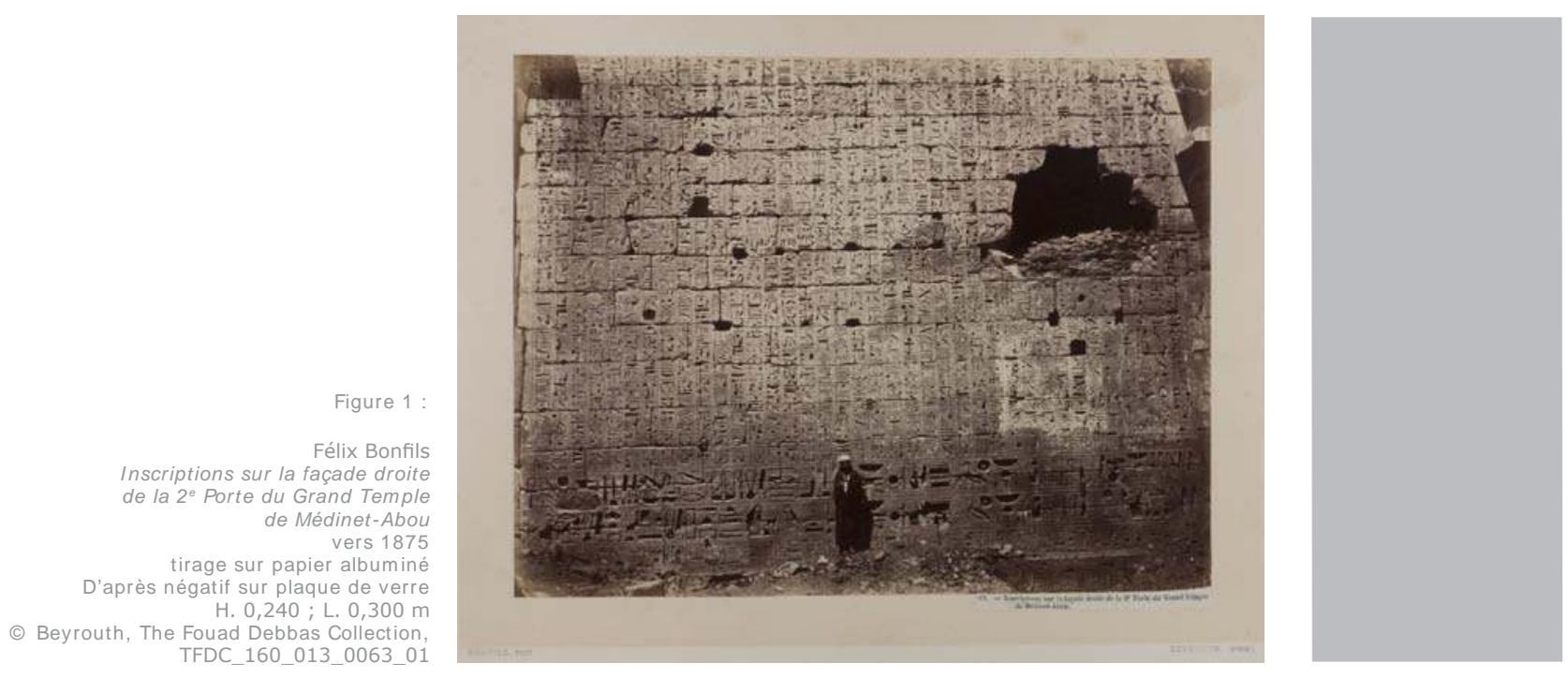

21. Bonfils, Inscriptions sur la façade droite de la $2^{e}$ Porte du Grand Temple de Médinet-Abou, TFDC_160_013_0063. http://eap.bl.uk/database/overview item.a4d?catld=197112: $r=292$ 22. Andrieux, Les célèbres statues de Memnon (Thèbes), TFDC 2152902

23. Bonfils, Temple d'Abou Simbel, TFDC 5030050084.

http://eap.bl.uk/database/overview item.a4d? catId=198488;r=17673

24. L. Gauthier art. cit. note 9, p. 5.

25. Cité par S. Moussa, «Rencontres en images. Regard du voyageur, regard du modèle », dans G. Beaugé, J.-F. Clément, L'image dans le monde arabe, Paris, CNRS, 1995, p. 227. G. Flaubert et M. du Camp voyagèrent entre 1849 et 1850 dans différents pays d'Orient, dont l'Égypte. 26. Voir par exemple I'album 159 de Bonfils.

http://eap.bl.uk/database/overview item.a4d?catId=197060;r=153 
Autre gageure pour les photographes-voyageurs, tenter de capturer l'intégralité du site semble être un but souvent recherché. Afin d'offrir un spectacle dans le sens le plus complet du terme, les photographes ont recours à des truchements. Félix Bonfils, avec une vue stéréoscopique de la colonne de Pompée d'Alexandrie, happe le regard vers le haut de la colonne qui semble toucher le ciel à l'extrémité du carton de montage ${ }^{27}$.

\section{Une mise en scène des exotismes}

Destination exotique de premier choix pour les Européens, l'Égypte est perçue comme un théâtre grandeur nature des Contes des Mille et Une Nuits ${ }^{28}$. De jour comme de nuit, les scènes de rues se mêlent aux récits d'un Orient idéalisé et enchanté. À travers la production photographique, l'évasion est recherchée, dans les paysages comme dans les portraits, tout en proposant des références communes auxquelles le public européen peut s'identifier.

Tout d'abord paysagé, l'exotisme est partout autour du Nil, dans le désert et ses représentations. Les éléments décoratifs très simples tels que le sable, un palmier et un peu d'eau suffisent à transporter l'homme dans une destination de rêve. La vision du Nil qui sortait de son lit au moment de la crue devait enchanter les Occidentaux, car de nombreux témoignages photographiques restituent ce spectacle naturel aujourd'hui annihilé par le barrage d'Assouan ${ }^{29}$. Les photographes n'hésitent pas à peupler ces " tableaux » de figurants soigneusement mis en scène au service d'un discours orientaliste ${ }^{30}$. Le chameau est notamment un ambassadeur de choix. Les photographes le placent près de l'eau du Nil, avec de préférence les silhouettes des pyramides reflétées à l'arrière-plan qui soutiennent le propos recherché31. Le public le découvre également servant d'attelage à une sakieh $^{32}$, paternité suggérée avec les scènes de labours occidentales. On sollicite ainsi l'imagination de l'observateur qui peut ressentir les bruits, l'odeur de poussière, et l'air circulant entre les feuilles des palmiers.

La photographie témoigne également d'une fascination pour la ville - vision même des contes des Mille et Une Nuits pour l'Occident ${ }^{33}$-avec la capture des formes de ses minarets, de ses dômes de mosquées, ou encore par les moucharabieh caractéristiques de ses fenêtres ; le jeu du « voir sans être vu " qu’ils impliquent ranime le mystère d'un Orient rêvé. Quelques rares photographies de villas ou palais ajoutent à cette dimension : la magnificence des édifices orientaux répond ainsi à la richesse architecturale des grandes demeures occidentales ${ }^{34}$.

Les photographes souhaitent également offrir à leurs clients des vues "à l'occidentale » de ces villes, dépeintes comme étant des centres commerciaux actifs grâce aux hôtels de Cook ou au passage de la ligne de chemin de fer, complétés par des promenades favorisées par une allée bordée d'arbres. Bonfils propose, notamment, des vues du débarcadère du port d'Alexandrie ${ }^{35}$, de la place des Consuls ${ }^{36}$ ou encore de la Rue de Choumbra au Caire ${ }^{37}$. Avec sa vue du Shepeards Hotel ${ }^{38}$, Le Caire s'identifie à une ville européenne, adaptée aux standards d'une clientèle internationale de plus en plus nombreuse, augmentée

27. Bonfils, Alexandrie, Colonne Pompée (Égypte), TFDC_0217_0002.

http://eap.bl.uk/database/overview item.a4d?catId $=196038 ; r=18467$

28. Ce recueil de contes populaires arabes d'origine persane et indienne fut traduit par A. Galland entre 1704 et 1717, traduction qui participa à sa diffusion en Europe.

29. Ce thème fut particulièrement photographié par $F$. Bonfils et les frères Zangaki, parmi d'autres.

30. Frères Zangaki, Arrosage des jardins au bord du Nil, TFDC_129_050_0386.

31. Frères Zangaki, Groupe des chameaux et Pyramides, TFDC_129_048_0214.

32. Hakim, Sakieh tournée par un chameau, TFDC 142 IMG8743.

33. Bonfils, Vue générale du Caire pris de l'Est, TFDC 401_11 1356. Publiée dans Les mille et une nuits, catalogue d'exposition de I'Institut du Monde Arabe, 2012 , p. 379.

http://eap.bl.uk/database/overview item.a4d?catld =197465: $r=1869$

34. C'est le cas de le vue stéréoscopique Kiosque situé dans le jardin du harem du prince Ismaïl-

Pacha à son palais de Guéziret, près du Caire (Égypte), TFDC_200_sn_IMG3133.

35. Bonfils, Débarcadère, Port d'Alexandrie, TFDC 5080010002.

http://eap.bl.uk/database/overview item.a4d?catId $=1 \overline{9} 852 \overline{5} ; r=28253$

36. Bonfils, Alexandrie, Place des Consuls, TFDC_508_002_0010.

http://eap.bl.uk/database/overview item.a4d?catId $=\overline{1} 985 \overline{2} 5 ; r=28253$

37. Fiorillo (attribué à), Rue de Choumbra, TFDC 139061 sn.

38. Bonfils, Caire, Shepeards Hôtel, TFDC_508_025_1123.

http://eap.bl.uk/database/overview item.a4d?catId $=198525 ; r=28253$ 
de la bonne société égyptienne. Cette transformation implique un commerce d'un genre nouveau, ce que Pierre Loti déplore quelques années plus tard au sujet de Louqsor : "Dans l'alignement pompeux du Winter Palace, des boutiques se succèdent : on y vend tout ce dont s'affublent les touristes : éventails, chassemouches, casques et lunettes bleues. En plus la bimbeloterie du Soudan : vieux couteaux de nègre, peaux de panthère et cornes de gazelles. Même les Indiens sont venus en foule à cette foire improvisée, apporter les étoffes du Radjapoute ou du Cachemire $^{39}$."

\section{La construction du portrait photographique}

Si la ville et les monuments sont largement mis à contribution par les photographes, la population locale participe également à l'illustration d'un certain exotisme. Il peut s'exprimer avec majesté à travers une véritable construction de portraits, galerie d'individus posant seuls ou en groupes. Les photographes utilisaient alors des toiles peintes tendues représentant généralement une végétation luxuriante qui tenaient lieu de décor. Les modèles, dépersonnifiés, y étaient insérés dans un espace correspondant au rendu recherché, par le biais d'accessoires tels que des bijoux, une chicha ou encore d'une activité jugée représentative du pays visitét ${ }^{40}$.
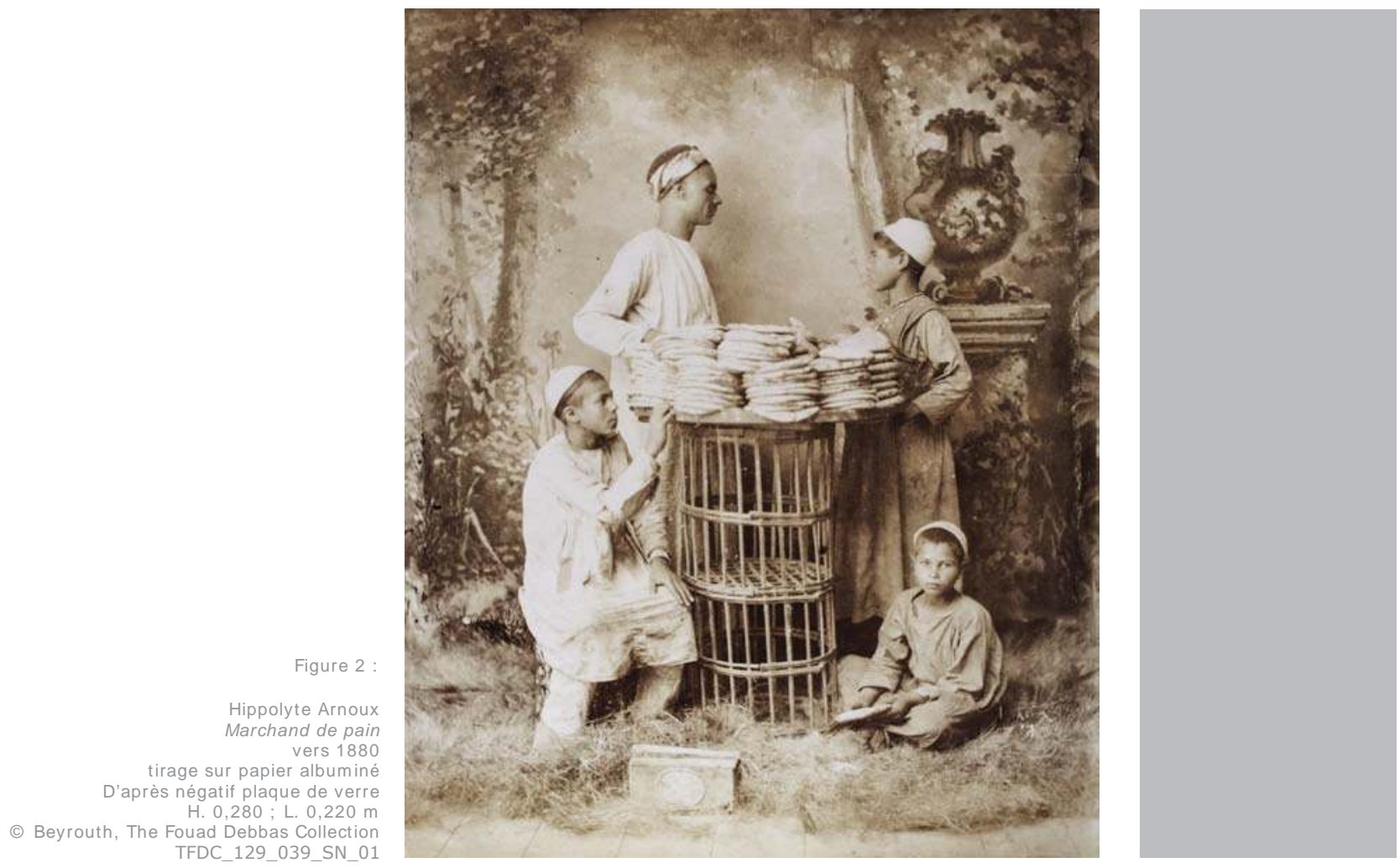

Toutefois, certaines incohérences trahissent la démarche du photographe dans sa recomposition du décor. Hippolyte Arnoux a produit une série de photographies dans laquelle il a recours à une toile de fond européanisant alors qu'il cherchait vraisemblablement à représenter la végétation luxuriante de l'Égypte. Or, les feuilles des arbres et l'effet forestier de ceux-ci ou encore la colonne antique gréco-romaine de l'arrière-plan de son vendeur de galettes ne trompent pas (fig. 2) ${ }^{41}$. L'effet escompté n'est pas atteint. Le sujet, certes, est égyptien, mais le photographe perd toute crédibilité en se trompant de fond ${ }^{42}$.

39. Pierre Loti, La Mort de Philæ, Paris, Calman-Lévy, 1908, p. 215.

40. L. Gauthier, art. cit. note 9 p. 7

41. H.Arnoux, Marchand de pain, TFDC_129_39_sn.

42. C'est également le cas avec la photographie d'un porteur d'eau, chargé de son outre pleine, mais passant devant un décor européanisant, dont on voit même la limite de la toile peinte servant de décor, là où Arnoux avait camouflé la transition entre la toile de fond et le sol. Voir TFDC_130_10_sn frères Zangaki (attribué à) Porteur d'eau dans une outre. 
Si quelques photographes, comme les frères Zangaki se sont, en apparence, spécialisés dans un type de scènes en plein-air, l'observation attentive de leurs clichés remet également en question la pertinence du contexte présenté. La Collection Fouad Debbas possède en effet un album ${ }^{43}$ où un décor unique sert de support à différentes photographies : la façade de moucharabieh en bois accolée à un mur de brique où se détache une porte en ogive est visible dans sa globalité sur une photographie intitulée Visite de cimetière (fig. 3) ${ }^{44}$; les mêmes moucharabieh servent de fond au Bazar des mandarines ${ }^{45}$, tandis qu'Une famille fellahine est disposée devant la porte en ogive ${ }^{46}$. Le décor est toujours le même, mais le sujet diffère, guidé par la légende qui trompe le public et supporte la mise en scène choisie.
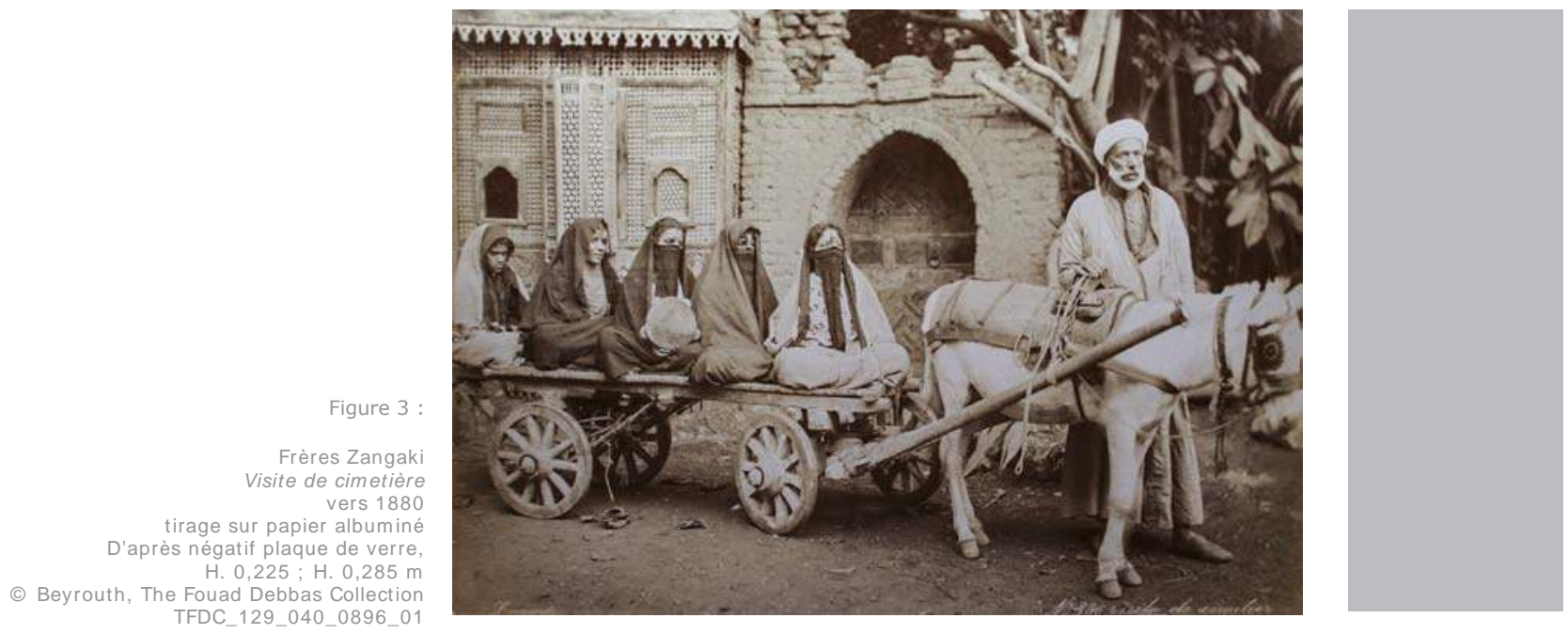

Vive source d'inspiration des écrivains et peintres orientalistes, la femme orientale n'est pas négligée dans la production des photographes commerciaux, qui participent à la construction de son image. Si les Shéhérazade ou autres Cléopâtre demeurent dans l'inconscient du voyageur, la photographie, par son illusion du réel, peut nuancer ces images fantasmées, et propose parfois une autre vision. Dans ce cas, les femmes sont décontextualisées : par groupes ou isolées, elles posent voilées, sur un fond uni et neutre qui les placent comme sujet principal. Le regard teinté de condescendance de l'Occident sur l'Orient se ressent dans certaines mises en scène : Bonfils place une mère sur un mulet avec son enfant, tandis que son époux tient l'animal par la bride. La femme regarde le sol, tandis que l'homme toise l'objectif ${ }^{47}$.

Les clichés qui permettent la diffusion des œuvres du musée de Boulaq n'échappent pas aux mises en scène du photographe, brouillant un aspect documentaire pourtant attendu dans ce contexte. Momies et statues peuvent être dressées contre une toile tendue pour faire office de fond, extraites de leur contexte muséographique habituel (fig. 4) ${ }^{48}$. D'autres effets théâtraux visent à décontextualiser le sujet. Bonfils ne s'intéresse pas au corps du pharaon Ramsès II mais décide de saisir exclusivement le visage de la momie, le présentant alors comme un portrait peint en médaillon ou un buste sculpté qui renverrait presque aux tondi italiens ${ }^{49}$.

43. TFDC_129. http://eap.bl.uk/database/overview item.a4d?catld $=196664: r=16827$

44. Frères Zangaki, TFDC $129040 \quad 0896$

45. Frères Zangaki, TFDC_129_45_0058.

46. Frères Zangaki, TFDC_129_56_0058.

47. Bonfils, Femme arabe et son enfant à baudet, TFDC_520_036_0733. Au sujet des femmes orientales dans la photographie, voir l'article de L. Gauthier, art. cit. note 9.

http://eap.bl.uk/database/ overview item.a4d?catld=198870; $r=27529$

48. Frères Zangaki (?), La statue de bois, TFDC_139_58_sn. Frères Zangaki, Momie de Séti ler, vue en pied, TFDC_139_60_sn.

49. Bonfils, Momies royales, tête de Ramsès I/ (19e dynastie) vue de face, TFDC_401_177_1182. http://eap.bl.uk/database/overview item.a4d?catld=197465: $r=1869$

Bonfils, Momies royales, tête de Ramsès II (19 ${ }^{e}$ dynastie) vue de profil, TFDC_401_178_1183. http://eap.bl.uk/database/overview item.a4d?catld $=197465 ; r=1869$ 

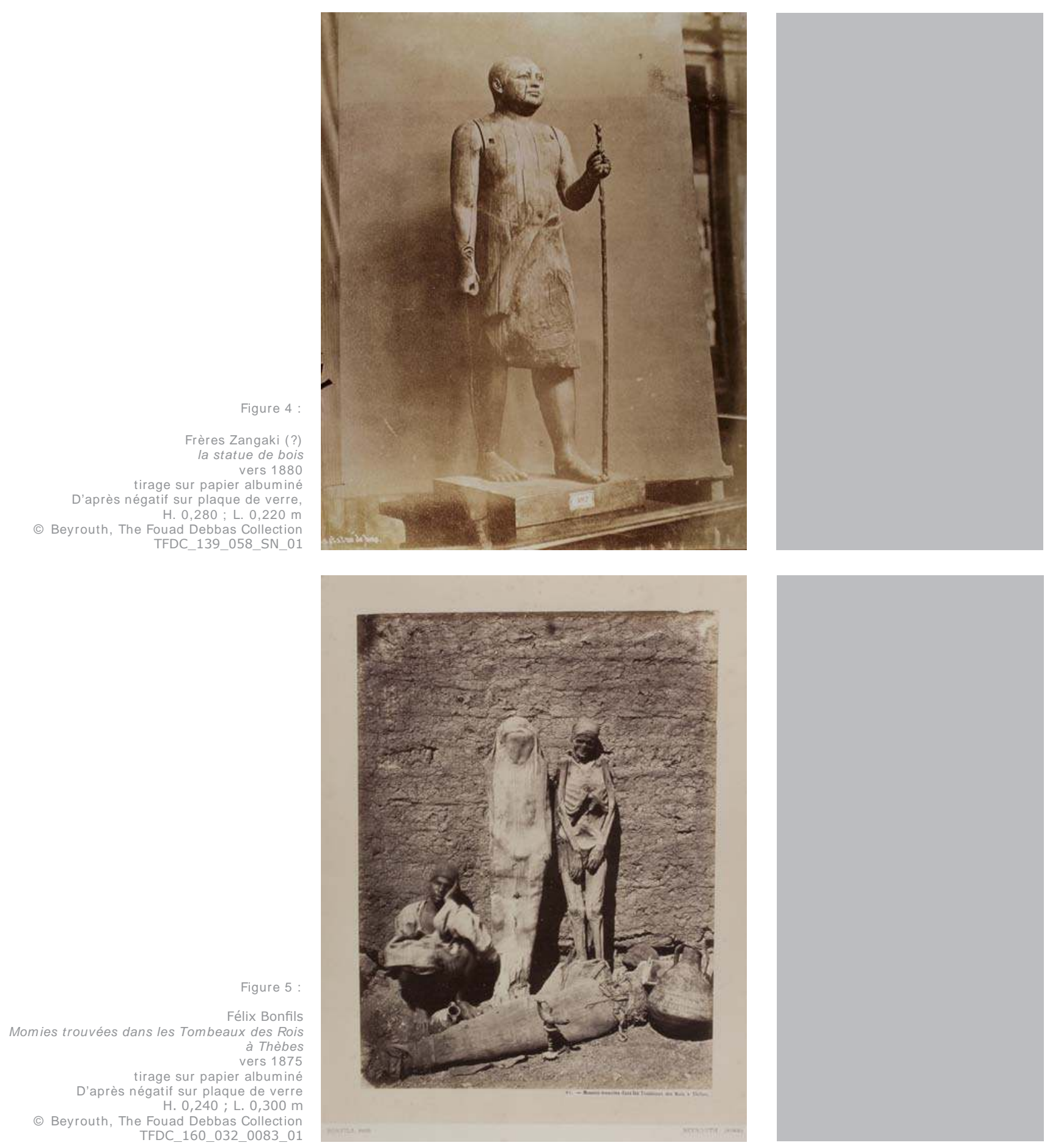

\section{L’Égypte, théâtre à ciel ouvert}

Par ses antiquités omniprésentes, l'Égypte est l'un des plus grands théâtres à ciel ouvert du XIX $X^{e}$ siècle et chaque photographe est désireux d'en capturer l'immensité désertique, les temples ou encore les momies, éléments très représentatifs de l'Égypte ancienne dans l'imaginaire européen. Ils poussent donc les artifices à leur paroxysme pour rendre le spectacle. Erreur technique du photographe Félix Bonfils ou démarche volontaire? Le figurant égyptien surgit de l'obscurité dans le temple de Karnak et donne vie au site sur certains clichés ${ }^{50}$. Dans la même série, Bonfils dispose deux momies royales contre un mur face à un fellah égyptien 
accroupi, la tête dans les mains. Le photographe cherche probablement ici à rendre l'idée d'une filiation entre l'Égypte d'hier et d'aujourd'hui ${ }^{51}$ (fig. 5).

On dénote également, parmi les photographies des monuments, un certain goût pour l'anecdote. Les tentations d'hier restent celles d'aujourd'hui, et bien que désormais prohibée, l'ascension de la pyramide de Chéops semble s'être imposée comme un rite de passage pour le voyageur. Gustave Flaubert en fait une description : " Les pierres qui à deux cents pas de distance semblent grandes comme des pavés n'en ont pas moins, les plus petites, trois pieds de haut. Généralement elles vous viennent à la poitrine - Nous montons par l'angle de gauche (celui qui regarde la pyramide de Chéphren). Les Arabes me poussent, me tirent - je n'en peux plus. C'est désespérant d'éreintement. Je m'arrête cinq ou six fois en route - Maxime est parti devant et va vite. Enfin j'arrive en haut ${ }^{52} . »$ L'ascension de la grande pyramide est immortalisée par de nombreux photographes (fig. 6). Comment ne pas sourire au spectacle cocasse de ces touristes tirés de bloc en bloc par les Égyptiens ${ }^{53}$ ? Du Camp - Maxime - complète : «Tous les bourgeois qui ont eu cette fortune de parvenir au sommet de la grande pyramide y ont gravé leur nom. Les pierres de la plate-forme disparaissaient sous ces ridicules monuments de bêtise et de vanité. Il y a des noms, de tous les pays et de toutes les langues ; j'ai vu là tous les croquis politiques, artistiques ou impurs qui ont si souvent égayé les murs de Paris. Les noms anglais abondent à côté de quelques noms de soldats français écrits à coups de baïonnette. Il y a des voyageurs qui ont fait de cela une enseigne, une réclame $[\ldots]^{54}{ }$. Le souci de Du Camp pour le patrimoine semble avoir fait peu d'émules et cette pratique fut également répandue parmi les photographes ; Bonfils lui-même laissa sa trace au Ramesseum, où l'on peut encore aujourd'hui lire: «F. Bonfils, 1875 ».
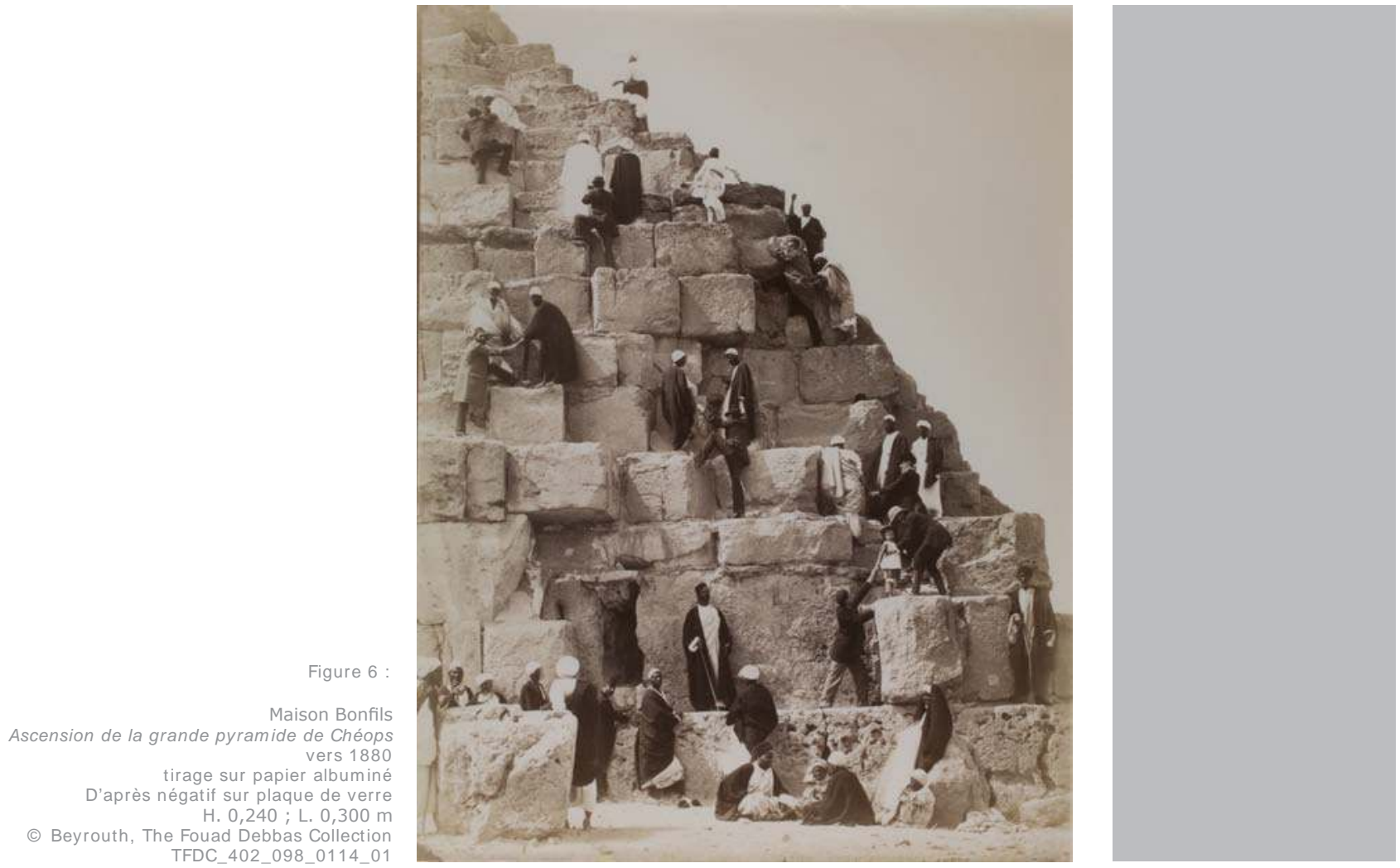

Bien que les intentions de Du Camp et des photographes commerciaux furent différentes, le caractère anecdotique ne doit pas faire oublier l'apport documentaire

51. Bonfils, Momies trouvées dans les Tombeaux des Rois, à Thèbes, TFDC_160_032_0083. http://eap.bl.uk/database/overview item.a4d?catld=197112: $r=292$

52. Voyage en Égypte, édition intégrale du manuscrit inédit et présentée par Pierre-Marc de Biasi, Grasset, Paris, 1991. Cité dans op.cit. note 14, p. 18

53. Maison Bonfils, Ascension de la grande pyramide de Chéops, TFDC 4020980114.

http://eap.bl.uk/database/overview item.a4d?catId=197536;r=19912

54. Maxime Du Camp, Le Nil, Paris, 1854, cité dans, A. D’Hooghe, op. cit. note 14, p. 19. 
non négligeable de ces clichés pour l'étude des monuments. À titre d'exemple, les nombreuses vues du sphinx de Chéphren témoignent de ses différentes phases de désensablement, et fixent l'état du site à un moment donné.

\section{Mœurs et usages}

Les photographes commerciaux, s'étant également donné pour mission d'enregistrer les mœurs et coutumes orientales, s'intéressent autant à la description des petits métiers traditionnels de l'Égypte, encore en activité pour certains, qu’à la pratique religieuse et aux festivités.

Bonfils, Arnoux ou encore les frères Zangaki produisent de nombreux clichés qui mettent en scène les activités quotidiennes de la population : du porteur d'eau ${ }^{55}$ au marchand de pain ${ }^{56}$ en passant par celle plus originale du vendeur de crocodiles ${ }^{57}$. L'artifice est toujours de mise, supporté par un décor construit et parfois inadéquat : Bonfils installe ses figurants dans la rue pour donner un contexte réaliste à sa photographie, tandis qu'Arnoux fait poser sa vendeuse de légumes devant une toile de fond luxuriante qui perturbe son discours ${ }^{58}$.

Les fêtes religieuses ou autres rites occupent une place plus rare mais qui pourrait être essentielle pour l'ethnologue, le sociologue de notre temps. Cependant, les photographes n'étant pas toujours familiers des traditions orientales interprètent, avec leur regard, le spectacle auquel ils assistent. Ainsi, Hammerschmidt, dans sa photographie au format « carte de visite " des Arabes faisant la prière ${ }^{59}$ donne à voir deux hommes, l'un agenouillé, les yeux fermés et les mains ouvertes tendues vers le ciel, s'apprêtant à embrasser le sol, et un autre, le dos courbé, les deux mains posées à terre encadrant son visage. Les paires de chaussures des deux hommes apparaissent au premier plan, et le tapis de prière sur lequel ils sont tous les deux assis donne le minimum d'informations nécessaires à la compréhension de l'Islam et de la prière qui est ici décomposée en deux temps. Toutefois, ces deux hommes partagent le même tapis et ne prient pas dans la même direction, ce qui rend la scène quelque peu incohérente : si les éléments rassemblés par le photographe sont intelligibles, il recompose son image en donnant sa propre interprétation de la prière.

D'autres photographes couvrent certaines festivités traditionnelles égyptiennes, tel le pèlerinage d'Aïd el-Kebir. La vue stéréoscopique Pilgrims of the Festival El-id-ed-Kebir Returning to Cairo ${ }^{60}$ plonge littéralement le spectateur au milieu de la foule. Pour rendre cette ferveur populaire, le photographe se place à un endroit stratégique d'où il saisit les files de pèlerins entrant et sortant de la ville, à pieds ou à dos d'âne. Au revers, un commentaire explique cette photographie stéréoscopique ; le texte est à destination des touristes et légende le spectacle "There is a close connection between feast and pilgrimages. At the great festivals in the Orient there are also great processions... ». Cette note rapproche le rite musulman des fêtes chrétiennes; il y a ici une volonté d'expliquer l'Orient à l'Occident en reprenant un système de pensée occidental et des référents communs.

Plus rares enfin, certains photographes osent la prise de vue de l'événement historique. Des tirages de Pascal Sebah témoignent du bombardement anglais d'Alexandrie en $1882^{61}$ où le fort d'Ada est la première place attaquée par la flotte britannique. Plusieurs photographies, toujours de Sebah, représentent de même la ville d'Alexandrie après son bombardement ${ }^{62}$. Mettant l'artifice de côté, le

55. F. Bonfils, Hamalis donnant à boire aux passants, TFDC_508_8548. http://eap.bl.uk/database/overview item.a4d?catId $=198525 ; r=28253$

56. H. Arnoux, Marchand de pain, TDFC_129_6798.

57. Cette activité est représentée chez différents photographes, comme les frères Zangaki. Marchand de crocodiles, TDFC_129_0589.

58. H. Arnoux, Vendeuse de légumes, TDFC_129_6859.

59. Arabes faisant la prière, TFDC CdvHammershmidt 54. II en est de même sur la photographie des frères Zangaki Musulmans en prière TDFC_129 05026706.

60. The Fine Art Photographer's Publishing $\mathrm{C}^{\circ}$, Pilgrims of the Festival El-id-ed-Kebir Returning to Cairo, Egypt, TFDC_204_9811.

61. Sebah, Les fortifications, TFDC_141_021_9450 ; Sebah, Vue du Fort Adda, pris du Fort Pharos, TFDC 1410210044

62. Sebah, Rüe Sésostris [après le bombardement], TFDC_141_IMG 9461 et Sébah, Rue Rosette [après le bombardement], TFDC_141_IMG 9465. 
photographe montre ce qu'il voit, de l'état de ruines des bâtiments à la population qui dégage les décombres laissés par les bombes.

De même, l'événement majeur que fut pour l'Égypte le percement et l'inauguration du canal de Suez fut particulièrement photographié. Arnoux, installé à Suez, fut à l'origine de la diffusion en Europe des tirages concernant le canal : les travaux et l'exploitation de la voie navigable sont systématiquement couverts par le photographe. Nombreux furent les touristes qui, de passage vers les Indes et l'Extrême-Orient profitèrent de l'escale à Port-Saïd pour visiter son atelier et acquérir quelques clichés ${ }^{63}$.

La production photographique du $\mathrm{XIX}^{\mathrm{e}}$ siècle est essentiellement liée aux attentes de la clientèle européenne autant avide de clichés souvenirs que d'images orientalistes. Répondant aux conventions qui s'appliquent à la peinture, la photographie s'impose par une restitution du réel. Toutefois, un certain nombre de photographes utilisent des artifices de mise en scène et les nombreuses possibilités techniques de leur médium pour rendre une illusion. À l'aube du XXe siècle, les photographes amateurs commencent à changer le discours des images du voyage en Égypte en annotant leurs photographies et en les plaçant dans des albums de souvenirs qui ressemblent à ceux de notre temps.

Malgré les artifices utilisés, ces clichés restituent la mémoire d'une époque - le XIX siècle -, d'un pays - l'Égypte -, et au sens plus large, d'une région. Telle fut la démarche de Fouad Debbas, pour qui préserver la mémoire du Moyen-Orient était au cœur de son activité de collectionneur. Si les monuments égyptiens sont relativement bien conservés, d'autres pays du Moyen-Orient ont aujourd'hui perdu une partie de leur patrimoine. De facto les albums et les tirages de la Collection Fouad Debbas constituent un témoignage inestimable de ces trésors disparus.

\section{Les auteurs}

Yasmine Chemali

Diplômée de l'École du Louvre en Arts de l'Islam et spécialisée en régie des ouvres, Yasmine Chemali vit et travaille à Beyrouth depuis 2011 où elle est responsable de la Collection Fouad Debbas, une collection privée de photographies anciennes. Membre de l'ICOM, de l'ICOMOS et de l'AIC, elle a reçu en 2013 une bourse du "Endangered Archives Programme » de la British Library afin de numériser, reconditionner et consolider les photographies de la Maison Bonfils au sein de la Collection Debbas. Elle est chargée de cours de conservation et de gestion des collections à Beyrouth. Yasmine Chemali a pour volonté de préserver le patrimoine visuel du Moyen-Orient en mettant en place des normes de professionnalisme et d'exigences internationales en matière de conservation préventive.

\section{Anne-Hélène Perrot}

Allocataire de recherche de l'École du Louvre pour l'année 2013-2014, Anne Hélène Perrot prépare un diplôme de $3^{\mathrm{e}}$ cycle en co-encadrement avec l'École doctorale de Lille qui a pour sujet : «Sépultures de la nécropole thébaine au Moyen Empire : relations entre les frises d'objets et le mobilier funéraire ». Chargée de Travaux dirigés devant les ouvres depuis 2011, et de cours en région, elle a collaboré avec le département des Antiquités égyptiennes du musée

63. Jean-Yves Tréhin, «H. Arnoux», dans François Pouillon (ed.), Dictionnaire des orientalistes de langue francaise, Paris, 2008, pp. 23-24. Albums photographiques du Canal de Suez (Port-Saïd, 1860-1890): Hyppolyte Arnoux. Photographe de l'union des mers, Paris, catalogue d'exposition, Centre historique des archives Nationales, Hôtel de Soubise, 6 nov. 1996-3 fév. 1997. 
du Louvre à l'occasion de deux expositions en 2011 et 2013 (Museum Lab L'offrande au temps des Égyptiens : une question de survie ; Rendre visite aux dieux : pèlerinage au temps de l'Égypte pharaonique). En 2014, elle a effectué une mission à la documentation du Centre d'Études Franco-Égyptien des temples de Karnak (CFEETK-Égypte) pour le projet Karnak. Depuis 2013, elle mène des recherches sur la partie égyptienne du fonds de la Collection Fouad Debbas en collaboration avec Yasmine Chemali, responsable de la collection. 